\title{
Characterization of intermittency in zooplankton behaviour in turbulence ${ }^{\star}$
}

\author{
François-Gaël Michalec ${ }^{1, a}$, François G. Schmitt ${ }^{2}$, Sami Souissi $^{3}$, and Markus Holzner ${ }^{1}$ \\ 1 Institute of Environmental Engineering, ETH Zurich, Stefano-Franscini-Platz 5, 8093 Zurich, Switzerland \\ 2 CNRS, Univ. Lille, Univ. Littoral Cote d'Opale, UMR 8187, LOG, Laboratoire d'Océanologie et de Géosciences, F62930 \\ Wimereux, France \\ 3 Univ. Lille, CNRS, Univ. Littoral Cote d'Opale, UMR 8187, LOG, Laboratoire d'Océanologie et de Géosciences, F62930 \\ Wimereux, France
}

Received 15 July 2015 and Received in final form 16 September 2015

Published online: 22 October 2015 - (C) EDP Sciences / Società Italiana di Fisica / Springer-Verlag 2015

\begin{abstract}
We consider Lagrangian velocity differences of zooplankters swimming in still water and in turbulence. Using cumulants, we quantify the intermittency properties of their motion recorded using three-dimensional particle tracking velocimetry. Copepods swimming in still water display an intermittent behaviour characterized by a high probability of small velocity increments, and by stretched exponential tails. Low values arise from their steady cruising behaviour while heavy tails result from frequent relocation jumps. In turbulence, we show that at short time scales, the intermittency signature of active copepods clearly differs from that of the underlying flow, and reflects the frequent relocation jumps displayed by these small animals. Despite these differences, we show that copepods swimming in still and turbulent flow belong to the same intermittency class that can be modelled by a log-stable model with non-analytical cumulant generating function. Intermittency in swimming behaviour and relocation jumps may enable copepods to display oriented, collective motion under strong hydrodynamic conditions and thus, may contribute to the formation of zooplankton patches in energetic environments.
\end{abstract}

\section{Introduction}

Calanoid copepods dominate the zooplankton community in marine and brackish environments. Due to their limited swimming abilities, these small planktonic organisms, which are about one millimetre in size when adult, interact generally very little with large features of the flow. Their behaviour is most significant when described at spatial scales that are on the order of a few centimetres or less, and at temporal scales on the order of a second [1]. At these small scales, behaviour allows copepods to capture preys [2], find mates [3] and escape predators [4].

Many species of calanoid copepods move by alternating periods of slow swimming with frequent relocation jumps. In filter-feeding copepods, the slow forward motion derives from the creation of feeding currents accomplished by the high-frequency vibration of the cephalic appendages [5]. In ambush-feeding species, where feeding and swimming are separate processes, copepods swim slowly to search for mates or foraging opportunities. Relocation jumps have been commonly observed in calanoid copepods, regardless of their feeding strategy, and result

\footnotetext{
* Contribution to the Topical Issue "Multi-scale phenomena in complex flows and flowing matter" edited by Luca Biferale, Massimo Cencini, Alessandra Lanotte and Mauro Sbragaglia.

a e-mail: michalec@ifu.baug.ethz.ch
}

in a sequence of small velocity bursts leading to an unsteady motion that appears rather erratic [6,7]. Consequently, the swimming behaviour of calanoid copepods in calm water can be considered as a generally steady motion, interrupted by frequent bursting events that result in comparatively high fluctuation levels for the velocity differences.

For levels of fluid turbulence characteristic of oceanic and estuarine habitats, small-scale flow velocity fluctuations and copepod maximal velocities are roughly comparable. Recent laboratory measurements have demonstrated the substantial contribution of active motion to the swimming dynamics of copepods in turbulence. Living animals swim faster and accelerate much stronger than their dead counterparts, even at moderate intensities of turbulence [8]. Conversely, turbulence cancels innate movement strategies and gender-specific differences in the degree of space occupation, two important behavioural features that determine how copepods interact with other organisms and how they explore their environment [9]. When motion strategies are not possible, vigorous swimming amid flow motion may allow copepods to maintain competitive advantage during interactions and thus, can improve survival and reproduction in a challenging environment. Swimming velocities achieved during steady propulsion in still water are low and do not contribute 
Table 1. Number of three-dimensional coordinates, number of trajectories and mean trajectory length (in frames) for the living copepods and the inert carcasses.

\begin{tabular}{lcccccc}
\hline & \multicolumn{2}{c}{ Coordinates } & \multicolumn{2}{c}{ Trajectories } & \multicolumn{2}{c}{ Length \pm s.d. } \\
\hline & Inert & Active & Inert & Active & Inert & Active \\
Calm water & - & 324801 & - & 253 & - & $1284 \pm 581$ \\
Turbulence & 1168685 & 1289810 & 2256 & 2601 & $518 \pm 357$ & $496 \pm 340$ \\
\hline
\end{tabular}

significantly to motion in turbulence. Copepods approximately drift with the flow unless executing a relocation jump. These swift movements, which are clearly visible even from visual observations [8], add up to the irregular nature of turbulent flows. In this study, we first test for intermittency in the swimming behaviour of copepods swimming freely in calm water. We then ask, using copepods swimming in turbulence, whether the intermittency signature of their self-induced motion amplifies that of the underlying flow.

One of the characteristic feature of fully developed turbulence is the intermittent nature of the velocity fluctuations [10]. According to Kolmogorov's scaling theory (K41), the high-order moments $\left\langle|\delta u(r)|^{p}\right\rangle$ of the Eulerian spatial velocity differences $\delta u(r)=u(x+r)-u(x)$ should scale as $r \zeta_{p}$ with $\zeta_{p}=p / 3$ in the inertial range, where the only relevant parameter is the energy flux $\epsilon$. Similarly, Lagrangian temporal velocity differences $\delta u(\tau)=$ $u(t+\tau)-u(t)$, also known as the Lagrangian structure functions, should scale as $D_{p}(\tau)=\left\langle|\delta u(\tau)|^{p}\right\rangle \sim(\epsilon \tau)^{\zeta_{p}}$ in the inertial range, with $\zeta_{p}=p / 2$. Experimental studies, however, have shown that both for Eulerian and Lagrangian quantities, the exponents $\zeta_{p}$ deviate strongly from their expected values [11-13]. This deviation, and the anomalous scaling of $\zeta_{p}$ are attributed to intermittency that provides corrections to the K41 predictions.

The exponents $\zeta_{p}$ are usually estimated for the range of scales where $D_{p}(\tau)$ displays a K41 scaling range with $\zeta_{2} \approx 1$ for the Lagrangian framework. The exponents, and their anomalous scaling, may also be more rigorously estimated via the extended self-similarity ansatz that requires to plot the structure functions of different orders against $D_{2}(\tau)[13,14]$. However, measurement of $\zeta_{p}$ has limitations because of finite Reynolds number and limited inertial range [15]. Such limitations affect measurements conducted with the structure functions and with the extended self-similarity method. We therefore consider another approach. Instead of studying the scale dependence for each moment, we focus on the moment dependence at a given scale, using cumulants. This method has been successfully used in the scaling turbulence framework to quantify the intermittency features of turbulent flows [1618] and here, we apply it to quantify intermittency in the motion of zooplankters.

\section{Methods}

\subsection{Presentation of the experimental data}

We have carried out laboratory experiments to track the motion of copepods swimming in turbulent flow [8]. The experimental setup and methodology are described in details in [8] and here, we briefly restate their main features. We produced turbulence in the laboratory using a setup that generates quasi-homogeneous and isotropic turbulence via counter-rotating disks [19]. The disks are $40 \mathrm{~mm}$ in diameter and smooth to prevent mechanical damage to the animals. We conducted three-dimensional Lagrangian particle tracking measurements using four synchronized cameras recording a $100 \mathrm{~Hz}$. Measurements were carried out within a $10 \mathrm{~cm} \times 10 \mathrm{~cm} \times 10 \mathrm{~cm}$ investigation volume centred in the middle of the aquarium and mid-way between the rotating disks. We conveyed 100 male copepods into the experimental vessel and recorded their behaviour for $5 \mathrm{~min}$ in still water and for $5 \mathrm{~min}$ under background flow motion. We conducted experiments twice. Because the mass density of calanoid copepods (density of approximately $1.05 \mathrm{~g} \mathrm{~cm}^{-3}$ ) is slightly higher than that of water [20], we conducted the same measurements on dead copepods to account for the effect of particle size and density [21]. We calibrated the cameras using images of a reference object with target points of known coordinates and we performed an additional dynamic calibration based on the images of moving particles [22]. We established correspondences between particle image coordinates and derived the three-dimensional positions of the moving particles by forward intersection, introducing their coordinates as unknowns in the augmented projection model $[23,24]$. We processed the image sequences and tracked living and dead copepods using an algorithm based on image and object space information [25].

The number of trajectories and number of threedimensional coordinates are indicated in table 1 . Turbulence parameters are given in table 2. The intensity of turbulence produced with our setup is comparable to characteristic values observed in coastal zones, tidal fronts and mega-tidal estuaries, three relatively energetic environments inhabited by calanoid copepods, where $\epsilon$ can reach $10^{-4} \mathrm{~m}^{2} \mathrm{~s}^{-3}[26,27]$. With the low $R_{\lambda}=35$ set in our measurements, we measured a second-order structure function that is similar in shape to those obtained at comparable [28] or very high Reynolds numbers [29] (fig. 1). We also observed a Lagrangian Kolmogorov constant $C_{0} \approx 2$ that agrees well with results from direct numerical simulations at $R_{\lambda}=43$, where $C_{0} \approx 2.5$ [28]. The initially sharper increase of the second-order structure function for the case of living copepods is due to their higher acceleration variance relative to that of the inert carcasses (fig. 1). Measurements were conducted with an ecologically representative species. We selected the estuarine calanoid copepod Eurytemora affinis because of its large distribution area which includes most temperate estuaries, its high importance in the trophic food web and the prevalence of turbulence in its habitat. 
Table 2. Turbulence parameters for the flow condition. $\epsilon$ is the turbulent energy dissipation rate. $\tau_{\eta}=(\nu / \epsilon)^{1 / 2}$ and $\eta=$ $\left(\nu^{3} / \epsilon\right)^{1 / 4}$ are the Kolmogorov time and length scales, respectively. $L$ is the integral length scale, estimated via the Eulerian velocity autocorrelation function. $T=L / u_{\mathrm{rms}}^{\prime}$ is the integral time scale. Finally, $R_{\lambda}$ is the Taylor Reynolds number.

\begin{tabular}{cccccc}
\hline$\epsilon\left(\mathrm{m}^{2} \mathrm{~s}^{-3}\right)$ & $\tau_{\eta}(\mathrm{s})$ & $\eta(\mathrm{mm})$ & $T(\mathrm{~s})$ & $L(\mathrm{~mm})$ & $R_{\lambda}$ \\
\hline $5.3 \times 10^{-5}$ & 0.13 & 0.4 & 0.8 & 6.2 & 35 \\
\hline
\end{tabular}

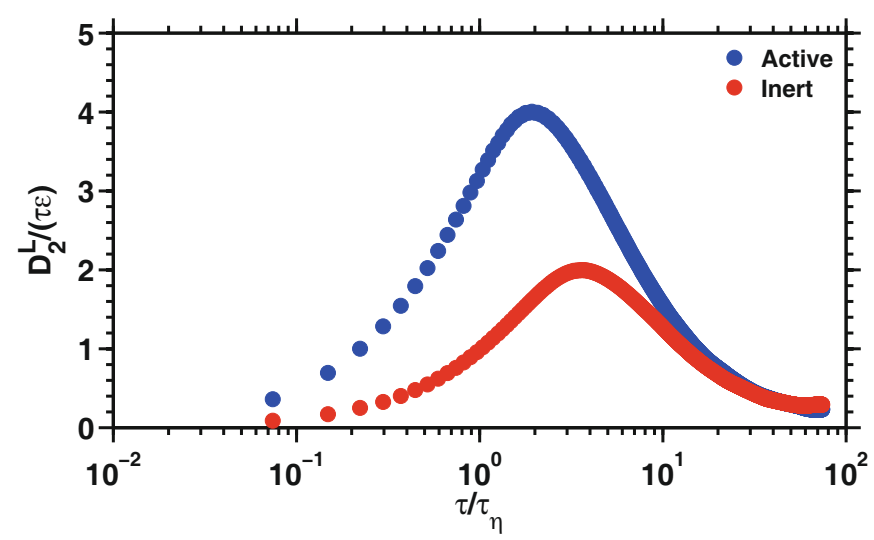

Fig. 1. Non-dimensionalised second-order structure function for active copepods (blue) and inert carcasses (red) in turbulent flow. The structure function displays a K41 scaling range with $\zeta_{2} \approx 1$ in the inertial range i.e. from 2 to 6 Kolmogorov time scales.

\subsection{Analysis of velocity fluctuations using cumulants}

We consider the variable $\delta u_{\tau}$ where $u$ is one component of the velocity vector and $\tau$ is the time increment along the trajectory of a particle. The moment generating function of the generator $g_{\tau}=\log \left(\left|\delta u_{\tau}\right|\right)$ is defined for each $\tau$ by $M_{g}(q)=\left\langle e^{g q}\right\rangle$. Its cumulant generating function is given by $\Psi(\tau)=\log \left(M_{g}(q)\right)=\log \left(\left\langle\left|\delta u_{\tau}\right|^{q}\right\rangle\right)$ [30]. The function $\Psi(\tau)$ is convex and, as a second characteristic function, can be developed via power series expansion, for each time increment $\tau$, using the cumulants [31]

$$
\Psi(q)=C_{1} q+\frac{1}{2 !} q^{2} C_{2}+\frac{1}{3 !} q^{3} C_{3}+\ldots=\sum_{p=1}^{+\infty} C_{p} \frac{q^{p}}{p !},
$$

where $C_{p}$ is the $p$-th cumulant. The first two cumulants are $C_{1}=\langle g\rangle=\left\langle\log \left(\left|\delta u_{\tau}\right|\right)\right\rangle$ and $C_{2}=\left\langle g^{2}\right\rangle-\langle g\rangle^{2}=\left\langle g^{2}\right\rangle-C_{1}^{2}$ and higher cumulants $C_{n}$ are polynomial functions of the moments $\left\langle g^{p}\right\rangle(1 \leq p \leq n)$. The theorem of Marcienkiewicz states that, if it exists, the development of eq. (1) is either infinite, or if finite, of a degree no higher than two [30]. In fact, the development of eq. (1) may not exist if $\Psi(q)$ is a non-analytic function. This occurs when $g$ is a stable process whose second-order moment (and hence its second-order cumulant) diverges. Stable distributions are sometimes referred to as Lévy-stable distributions and correspond to a generalization of the Gaussian law [32]. The main parameter is the index $\alpha$ bounded between 0 and 2, the upper bound corresponding to the normal distribution. Log-stable approximations for turbulence intermittency correspond to a non-analytic scaling moment function [33]. In this case, instead of eq. (1), we have the relation $[17]$

$$
\Psi(q)=C_{1} q+C_{\alpha} q^{\alpha},
$$

where $0 \leq \alpha \leq 2$ is the index of the stable process and $C_{\alpha}$ is the non-analytical cumulant. When $\alpha=2$ the generator is a Gaussian process and the variable $\delta u_{\tau}$ follows a lognormal distribution. Because of non-analycity, there are only two cumulants in the development of eq. (1). To check the suitability of log-stable approximation for our analysis, we consider the function

$$
\Phi(q)=\Psi(q)-C_{1} q
$$

For a stable law, $\Phi(q)$ should be proportional to $q^{\alpha}$. Using trajectories of active and inert copepods, we estimate the functions $\Phi(q)$ for moments up to 6 and for time increments up to $10 \mathrm{~s}$. Using a best fit, the slopes of the straight lines after log-transformation give directly the values of the exponent $\alpha$ for all scales, and the $y$-intersects of these linear trends give the values of $C_{\alpha}[17,18]$.

\section{Results}

We show in fig. 2 A the probability density function of the velocity increment $\delta u_{\tau}$ for copepods swimming in calm water, at different values of $\tau$. The swimming behaviour is characterized by a large proportion of very small velocity increment values, and by stretched exponential tails. The large proportion of small velocity differences derives from the slow swimming behaviour of E. affinis; in calm conditions, copepods most often cruise steadily through the water. Relocation jumps explain the exponential tails. Figure $2 \mathrm{~B}$ shows the probability density function of $\delta u_{\tau}$ for living copepods and inert carcasses in turbulence. The parameterization curve of [34], used to fit the experimental data of particle acceleration at high Reynolds number, is shown for comparison. This stretched exponential fit follows our experimental data well. Surprisingly, we find that velocity differences of living and inert particles in turbulence collapse when normalized by their variance. A similar shape at small velocity differences was not unexpected, because the turbulent transport dominates over slow cruising speeds. However, also the tails of the probability density functions collapse rather well, which suggests that relocation jumps and strong fluid acceleration produce a similar intermittency.

As expected for second characteristic functions, the experimental curves of $\Psi(q)$ are convex and converge rapidly to a limit function as the separation time increases (fig. 3). The fits obtained via eq. (2) agree well with empirical data, especially for values of $q<5$, except for active animals in still water for which $\alpha$ and $C_{\alpha}$ have been estimated with $0.75 \leq q \leq 6$. We also show in fig. 3 the function $\log (\Phi(q))$ vs. $\log (q)$ for different values of $q$ and 

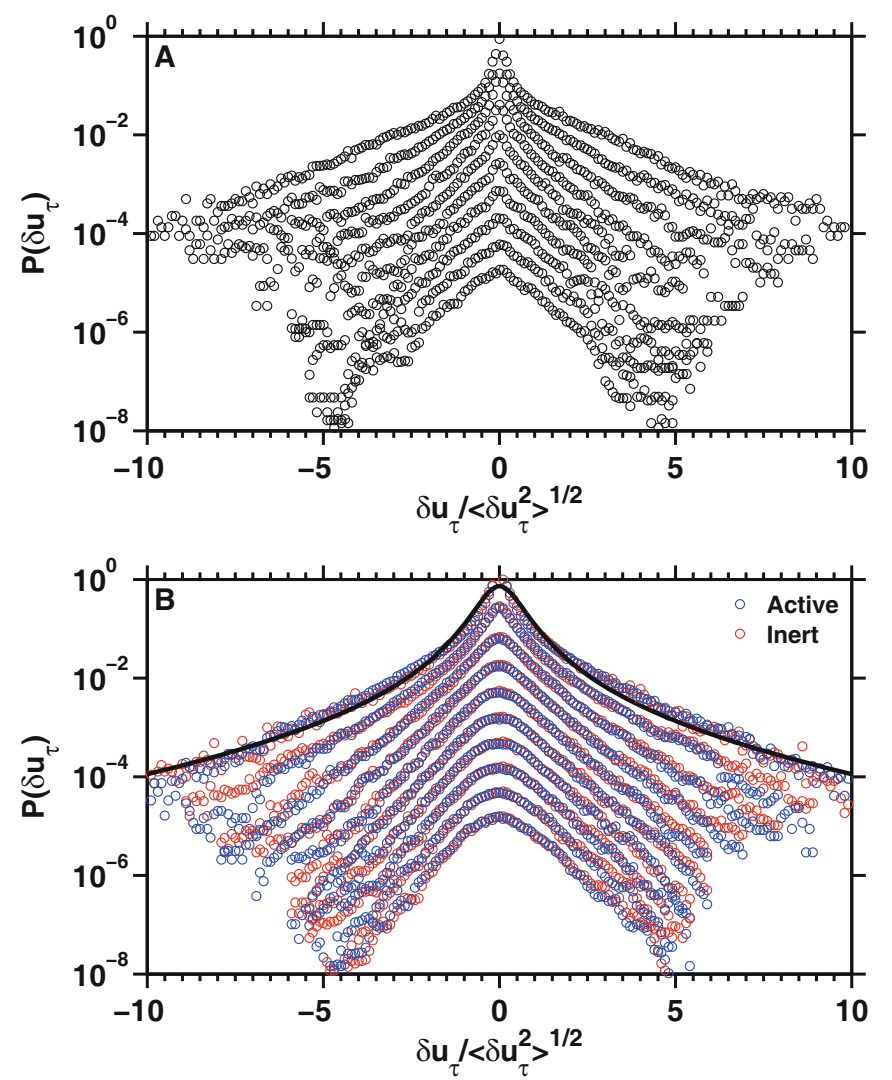

Fig. 2. Probability density functions of the normalized increments $\delta u_{\tau} /\left\langle\delta u_{\tau}^{2}\right\rangle^{1 / 2}$ for different values of $\tau$ ranging from $0.01 \mathrm{~s}$ to $4.5 \mathrm{~s}$ (from top to bottom; curves are vertically shifted) for living copepods in still water (black) (A) and for living (blue) and inert (red) copepods in turbulence (B). The stretched exponential fit presented in [34] and obtained with tracer particles at $R_{\lambda}=970$ is indicated in (B) as a solid black line, for comparison. Our experimental data agrees well with the stretched exponential fit, despite a much lower $R_{\lambda}$.

for time increments ranging from $0.01 \mathrm{~s}$ to $1 \mathrm{~s}$. The straight lines obtained for a wide range of $q$ confirm the validity of our non-analytic framework, and agree with the logstable model. Using a best fit, the slope of these straight lines are estimated for all scales, giving directly the exponents $\alpha$ in eq. (2), and their $y$-intersect gives the values of the non-analytical cumulant $C_{\alpha}(\tau)$. Figure 4 A shows the first cumulant $C_{1}$ for living animals with and without flow, and for inert animals with flow. For the turbulence case, $C_{1}$ is proportional to $\tau$ in the inertial range, which has been estimated in our measurements between 2 and 6 Kolmogorov time scales (i.e. less than 1s) (fig. 1). Differences are clearly visible between active and inert copepods in turbulence for separation times below $1 \mathrm{~s}$. They disappear at larger $\tau$, suggesting that at large separation times, flow motion is the main contributor to the intermittency features of the motion. We show in fig. $4 \mathrm{~B}$ the nonanalytical cumulant $C_{\alpha}(\tau)$. Here again, $C_{\alpha}(\tau)$ is proportional to $\tau$ in the inertial range, and differences are visible between dead and living animals only at very short separation times (up to $0.2 \mathrm{~s}$ ), above which $C_{\alpha}(\tau)$ reflects the global dynamics of the flow. We quantify the uncertainty of our estimation by computing $C_{\alpha}(\tau)$ for different scaling ranges: we find a mean standard deviation of 0.03 and 0.04 for living and inert copepods in turbulence, respectively, and a mean standard deviation of 0.3 for living animals in still water. Figure $4 \mathrm{C}$ shows the exponent $\alpha$. The values are relatively independent of the scale, except for living animals in still water at large separation times. Computing $\alpha$ for different scaling ranges gives a mean standard deviation of 0.07 for living copepods in turbulence, 0.08 for inert carcasses in turbulence, and 0.6 for living copepods in still water. The larger value observed for living animals without turbulence suggests a larger departure from the log-stable model, or that more data is needed for the statistics to converge better. We can however estimate mean values: we find $\alpha=1.62 \pm 0.07$ for living animals in still water, $\alpha=1.67 \pm 0.02$ for living animals in turbulence and $\alpha=1.66 \pm 0.04$ for inert copepods in turbulence. These mean values are approximately comparable between living and inert copepods in turbulence, and also for living animals without flow. For the latter however, the slightly lower exponent may indicate a stronger departure from Gaussian law. For active and inert copepods in turbulence and unlike $C_{\alpha}(\tau)$, the exponent $\alpha$ does not vary substantially in the inertial range. For living animals in still water, the variation of both $\alpha$ and $C_{\alpha}$ for $0.1 \leq \tau \leq 2$ points to the existence of two behaviours at small and large separation times, with a transition region. However, the uncertain behaviour of $\alpha$ for $\tau \geq 3$ suggests that more data is needed to better characterize changes in its statistical properties.

\section{Discussion}

We have considered here Lagrangian velocity measurements on the motion of calanoid copepods in calm water and in turbulent flow. By using cumulants, we precisely characterized the contribution of each scale to velocity fluctuations and thus, velocity intermittency at different time scales. We first aimed at quantifying the intermittency signature of living copepods swimming in still water. We then assessed the relative contribution of self-induced motion and advection due to flow to the intermittency features of animals swimming in turbulence. The following main points emerge from our analysis:

- Swimming zooplankton particles in still and turbulent flow belong to the same intermittency class that can be modelled by a log-stable model with non-analytical cumulant generating function and with a characteristic exponent $\alpha=1.65 \pm 0.15$.

- The shape of the normalised probability density function of the velocity increment bears the same intermittency signature for living and inert copepods in turbulence. That is not to say, however, that copepods behave as passive particles in turbulent flow. The energy dissipation rate in our measurements (i.e. $5.3 \times 10^{-5} \mathrm{~m}^{2} \mathrm{~s}^{-3}$ ) is representative of moderate turbulence in oceanic and estuarine environments. At this 

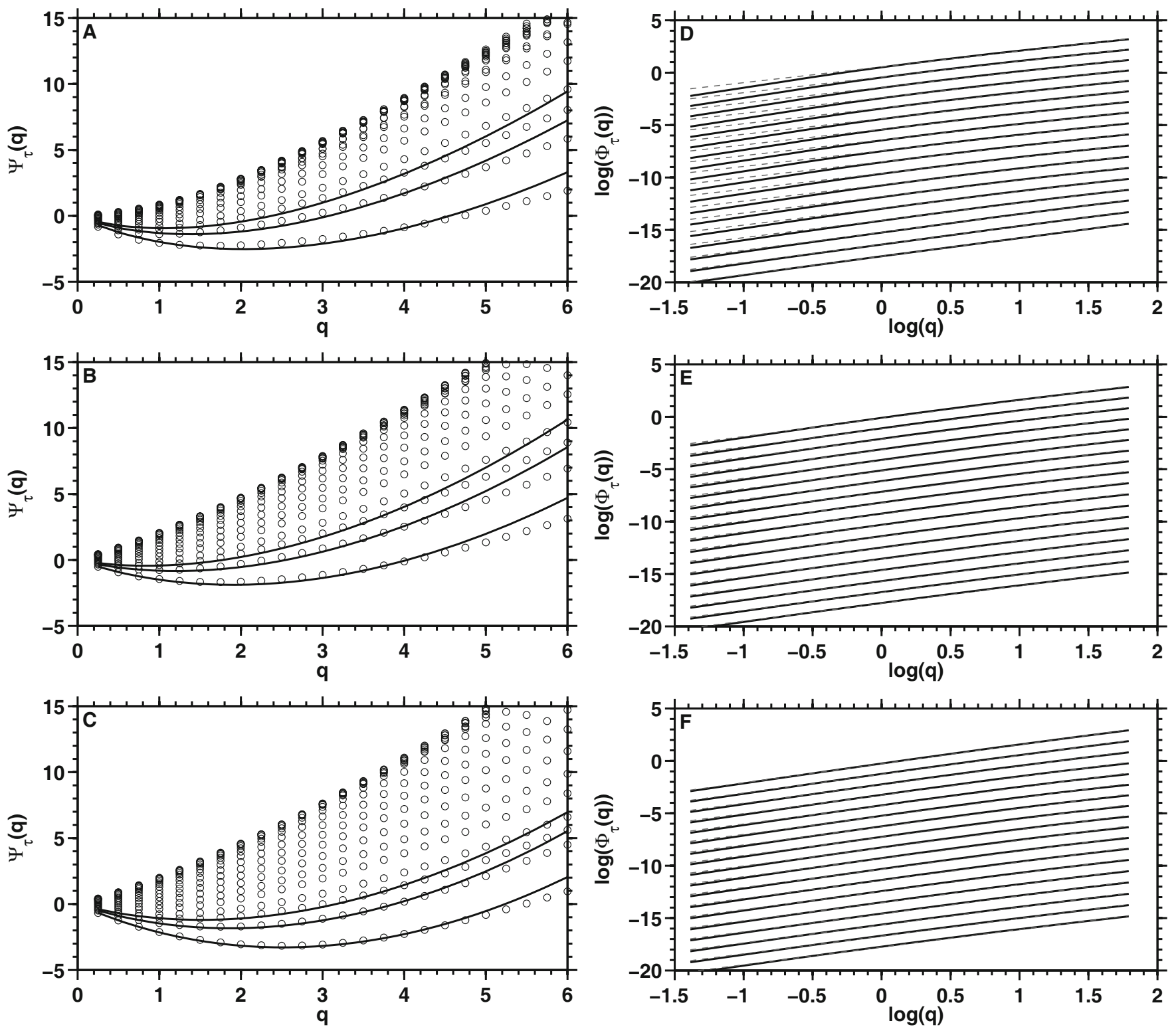

Fig. 3. The second characteristic function $\Psi(q) v s$. $q$ for living copepods in still water (A), for living copepods in turbulence (B) and for inert copepods in turbulence $(\mathrm{C})$, for time increments logarithmically distributed between $0.01 \mathrm{~s}$ and $3 \mathrm{~s}$ (from bottom to top). Dots indicate empirical values; continuous lines are obtained via eq. $(2) \cdot \log (\Phi(q)) v s . \log (q)$ for living copepods in still water (D), for living copepods in turbulence (E) and for inert copepods in turbulence (F), for time increments logarithmically distributed between $0.01 \mathrm{~s}$ and $3 \mathrm{~s}$ (from top to bottom; curves are vertically shifted for clarity). Black continuous lines show experimental values, grey dashed lines correspond to straight lines with a slope of $\alpha$.

intensity of turbulence, copepods can accelerate much more strongly than the flow: we measured that dimensional accelerations are stronger for living copepods than for inert carcasses [8]. Only when velocity differences are normalized by their respective variance do the probability density functions overlap.

- Differences between active animals and inert carcasses in turbulent flow are clearly visible at short time scales when considering the first cumulant $C_{1}(\tau)$ and the non-analytical cumulant $C_{\alpha}(\tau)$. These differences come from sharper accelerations due to rapid and frequent relocation jumps. At longer time scales, the intermittency signature of living copepods in turbulence reflects that of the turbulent flow.

Intermittency in flying or swimming small animals is not well studied, despite its ecological importance. In swarming midges, the probability density function of the acceleration shows very heavy tails, and the spatial structure of the acceleration - toward the centre of the swarm - helps to keep the swarm intact [35]. In 

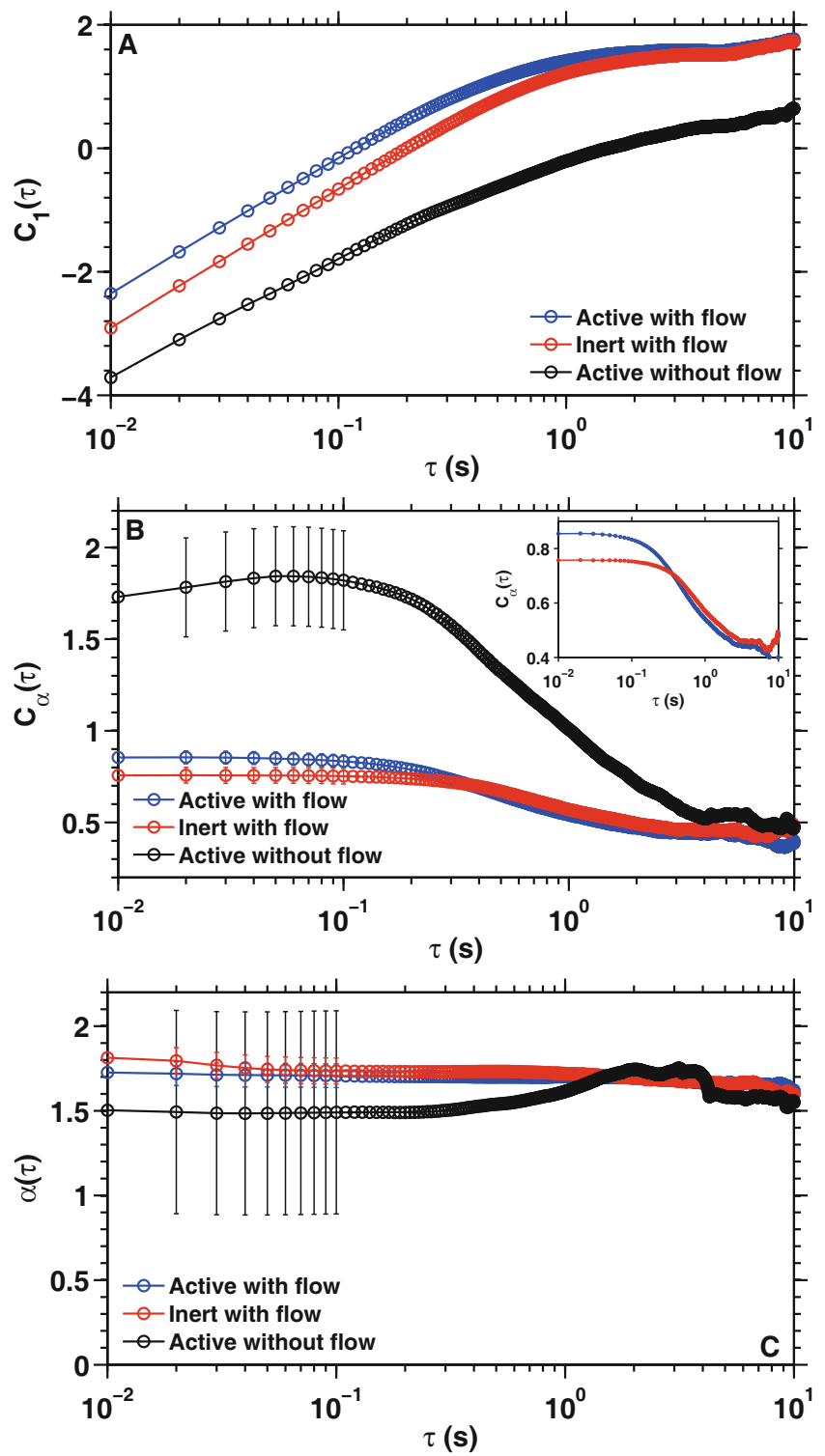

Fig. 4. The first cumulant $C_{1}=\left\langle\log \left(\left|\delta u_{\tau}\right|\right)\right\rangle$ estimated at different values of the separation time $\tau$ for living animals swimming in calm water (black), for living animals swimming in turbulence (blue) and for inert copepods in turbulence (red) (A). Values of the non-analytical cumulant $C_{\alpha}(\tau)$ at different values of $\tau$. Because of differences in the scaling range between flow and no-flow conditions, an inset shows $C_{\alpha}(\tau)$ for the living and inert copepods in turbulence, over a more suitable range (B). Values of the exponent $\alpha$ estimated at different values of $\tau(\mathrm{C})$. Panels (B) and (C) show the mean standard deviation obtained by computing $C_{\alpha}(\tau)$ and $\alpha(\tau)$ for different scaling ranges. The mean standard deviation is indicated for a limited number of points only, for clarity.

copepods, intermittent behaviour is necessary to maintain swimming efficiency in turbulent flow [8], because swift movements may help copepods to escape from predators and find mates [36,37]. Eurytemora affinis often dominates the estuarine zooplankton community in the low salinity zone, despite very high dissipation rates and currents with mean speed beyond
$2 \mathrm{~m} \mathrm{~s}^{-1}$ [38]. This species displays active vertical migrations that allow the maintenance of its population along the estuarine salinity gradient [39]. This behaviour has been found to depend on swimming capabilities. Adults and late-stage copepodites migrate to the bottom layer, both at ebb tide and during the late rising tide, whereas nauplii are transported as passive particles in the turbidity maximum zone [40]. The intermittent behaviour of adult copepods, and the velocities achieved during relocation jumps, may explain the ability of these small animals to display large-scale directed motion in turbulent environments [41,42].

In conclusion, our approach based on cumulant scaling provides a reliable alternative to methods based on structure functions and extended self-similarity, since it relates to statistical analysis at a given scale, whereas the accurate determination of adequate intermittency models using structure functions is often constrained by the imperfect scale-invariance of the statistics. The methods is also useful for comparison between active and passive particles. We have shown that the log-sable model applies very well, with a characteristic exponent valid for all scales. These properties can be used for zooplankton behaviour modelling, with useful ecological applications such as plankton-turbulence coupling or feeding efficiency of fish larvae.

\section{Author contribution statement}

F.-G. M. and M. H. designed the experiments. S. S. provided the test organisms. F.-G. M. carried out the measurements, performed data analysis and drafted the manuscript. S. S., F. G. S and M. H. participated in data analysis and helped draft the manuscript. All authors gave final approval for publication.

This work was supported by Swiss National Science Foundation (SNSF) grant number No. 144645 and ETH grant No. 2914-1, and is a contribution to the bilateral agreement between ETH Zurich and Lille 1 University. We thank the organisers of the FloMat2015 conference for an inspiring meeting.

\section{References}

1. T. Kiørboe, Oecologia 155, 179 (2008).

2. S. Kjellerup, T. Kiørboe, Biol. Lett. 8, 438 (2012).

3. M. Uttieri, A. Nihongi, M.G. Mazzocchi, J.R. Strickler, E. Zambianchi, J. Plankton Res. 29, i17 (2007).

4. B.J. Gemmell, J. Sheng, E.J. Buskey, Proc. Natl. Acad. Sci. U.S.A. 110, 4661 (2013).

5. T. Kiørboe, H. Jiang, R.J. Gonçalves, L.T. Nielsen, N. Wadhwa, Proc. Natl. Acad. Sci. U.S.A. 111, 11738 (2014).

6. F.G. Schmitt, L. Seuront, Physica A 301, 375 (2001).

7. H. Jiang, T. Kiørboe, J. R. Soc. Interface 8, 1090 (2011).

8. F.G. Michalec, S. Souissi, M. Holzner, J. R. Soc. Interface 12, 20150158 (2015).

9. G. Bianco, P. Mariani, A.W. Visser, M.G. Mazzocchi, S. Pigolotti, J. R. Soc. Interface 11, 20140164 (2014). 
10. U. Frisch, Turbulence: The legacy of A. N. Kolmogorov. (Cambridge University Press, Cambridge, UK 1995).

11. F. Anselmet, Y. Gagne, E.J. Hopfinger, R.A. Antonia, J. Fluid Mech 140, 63 (1984).

12. N. Mordant, P. Metz, O. Michel, J.F. Pinton, Phys. Rev. Lett. 87, 214501 (2001).

13. H. Xu, M. Bourgoin, N.T. Ouellette, E. Bodenschatz, Phys. Rev. Lett. 96, 024503 (2006).

14. R. Benzi, S. Ciliberto, R. Tripiccione, C. Baudet, F. Massaioli, S. Succi, Phys. Rev. E 48, R29 (1993).

15. Y. Huang, L. Biferale, E. Calzavarini, C. Sun, F. Toschi, Phys. Rev. E 87, 041003(R) (2013).

16. L. Chevillard, S.G. Roux, E. Lévêque, N. Mordant, J.F. Pinton, A. Arnéodo, Phys. Rev. Lett. 95, 064501 (2005).

17. F.G. Schmitt, in Nonlinear Science, Complexity edited by A.C.J. Luo, L. Dai, H.R. Hamidzadeh (World Scientific; New Jersey, 2007), p.p. 240-246.

18. F.G. Schmitt, Y. Huang, Z. Lu, Y. Liu, N. Fernandez, J. Marine Syst. 77, 473 (2009).

19. K. Hoyer, M. Holzner, B. Lüthi, M. Guala, A. Liberzon, W. Kinzelbach, Exp. Fluids 39, 923 (2005).

20. T. Knutsen, W. Melle, L. Calise, J. Plankton Res. 23, 859 (2001).

21. H. Xu, E. Bodenschatz, Physica D 237, 2095 (2008).

22. A. Liberzon, B. Lüthi, M. Holzner, S. Ott, J. Berg, J. Mann, Physica D 241, 208 (2012).

23. H.G. Maas, A. Gruen, D. Papantoniou, Exp. Fluids 15, 133 (1993).

24. N.A. Malik, T. Dracos, D.A. Papantoniou, Exp. Fluids 15 279 (1993).

25. J. Willneff, A. Gruen, in Proceedings of the 9th International Symposium on Transport Phenomena and Dynamics of Rotating Machinery. Honolulu, HI, USA; (2002).

26. H. Peters, J. Phys. Oceanogr. 33, 1967 (2003).
27. H.L. Pécseli, J.K. Trulsen, Ø. Fiksen, Prog. Oceanogr. 85, $171(2010)$.

28. P.K. Yeung, S.B. Pope, B.L. Sawford, J. Turbul. 7, (2006) DOI: $10.1080 / 14685240600868272$.

29. L. Biferale, E. Bodenschatz, M. Cencini, A.S. Lanotte, N.T. Ouellette, F. Toschi et al., Phys. Fluids 20, 065103 (2008).

30. C.W. Gardiner, Handbook of stochastic methods, 3rd edition (Springer, Berlin, 2004).

31. W. Feller, An introduction to probability theory and its applications 3rd edition (John Wiley \& Sons, New York, 1971).

32. G. Samorodnitsky, M.S. Taqqu, Stable non-Gaussian random processes (Chapman \& Hall/CRC, New York, 1994).

33. D. Schertzer, S. Lovejoy, F.G. Schmitt, Y. Chigirinskaya, D. Marsan, Fractals 05, 427 (1997).

34. A. La Porta, G.A. Voth, A.M. Crawford, J. Alexander, E. Bodenschatz, Nature 409, 1017 (2001).

35. D.H. Kelley, N.T. Ouellette, Sci. Rep. 3, 1073 (2013).

36. T. Kiørboe, A. Andersen, V.J. Langlois, H.H. Jakobsen, J. R. Soc. Interface 7, 1591 (2010).

37. D. Adhikari, E.K. Longmire, Meas. Sci. Technol. 24, 024011 (2013).

38. D. Devreker, S. Souissi, J.C. Molinero, D. Beyrend-Dur, F. Gomez, J. Forget-Leray, Estuar Coast Shelf Sci. 89, $245(2010)$.

39. C.A. Morgan, J.R. Cordell, C.A. Simenstad, Marine Biol. 129, 309 (1997).

40. F.G. Schmitt, D. Devreker, G. Dur, S. Souissi, Ecol. Res. 26, 773 (2011).

41. W.J. Kimmerer, J.R. Burau, W.A. Bennett, Estuaries 25, 359 (2002).

42. A. Genin, J.S. Jaffe, R. Reef, C. Richter, P.J.S. Franks, Science 308, 860 (2005). 